\title{
CONIVEAU FILTRATION AND MIXED MOTIVES
}

\author{
FRÉDÉRIC DÉGLISE
}

\begin{abstract}
We introduce the motivic coniveau exact couple of a scheme, in the framework of mixed motives, whose property is to universally give rise to coniveau spectral sequences through realizations. The main result is a computation of its differentials in terms of residues and transfers of mixed motives, with a formula analog to the one defining the Weil divisor of a rational function. We then show how to recover and extend classical results of Bloch and Ogus for motivic realizations.
\end{abstract}

\section{INTRODUCTION}

The coniveau filtration is deeply rooted in the main conjectures on algebraic cycles, the ones of Hodge and Tate, as stated by Grothendieck in Gro69 and Gro68. This filtration was first define on De Rham cohomology by Grothendieck as the abutment of a spectral sequence deduced from the Cousin resolution of coherent sheaves (see Gro66, footnote (8), p. 356-357]). In fact, as it appears in Har66, chap. IV], Cousin resolutions can be understood as the lines in the $E_{1}$ term of a suitable sheafified spectral sequence for cohomology with coefficients in a coherent sheaf. This kind of spectral sequence is now called coniveau spectral sequence after the landmarking work of Bloch and Ogus [BO74].

As higher algebraic K-theory was rising, Gersten extended the preceding considerations to the case of K-theory ending in the celebrated Gersten conjecture 1 At that time, Bloch discovered the connection of the coniveau spectral sequence in Ktheory with algebraic cycles, formulating what is now called, after Quillen, Bloch's formula. In fact this formula is a consequence of the Gersten conjecture, as showed by Quillen as a corollary of his proof of the conjecture in the equicaracteristic case ([Qui73, 5.19]). It is important for us to recall that Quillen deduces this fact from the identification of some of the differentials in the $E_{1}$-term of the coniveau spectral sequence with the classical divisor class map, associating to a rational function its Weil divisor (Qui73, proof of 5.14]). The purpose of this article is to extend this computation in the theory of Voevodsky's motives.

Motivated by the circle of ideas around the Gersten conjecture, Rost introduced in [Ros96] a notion of local system, called cycle modules, which gives a theoretical framework to define a generalized divisor class map for certain cycles with coefficients. The primary example of a cycle module is the well-known Milnor K-functor $K_{*}^{M}$. As an illustration, we recall the definition of the generalized divisor class map for a normal algebraic connected $k$-scheme $X, k$ being a field. In this case, any codimension 1 point $x$ of $X$ corresponds to a discrete valuation $v_{x}$ of the function

\footnotetext{
Date: June 2011.

Partially supported by the ANR (grant No. ANR-07-BLAN-042).

${ }^{1}$ Recall this conjecture can be phrased by saying that the Cousin complex of the unramified K-theory sheaf over a regular scheme is a resolution: see [Ger73, Problem 10, p. 46]. Following Har66 Def. p. 238], one also says this sheaf is Cohen-Macaulay.
} 
field $E$ of $X$, whose residue field $\kappa(x)$ is the residue field of $x$ in $X$. To the valued field $\left(E, v_{x}\right)$ is associated the so called tame symbol map (cf [Mil70, 2.1]):

$$
\partial_{v_{x}}: K_{*}^{M}(E) \rightarrow K_{*}^{M}(\kappa(x))
$$

an homogeneous morphism of graded abelian groups of degree -1 which in degree 1 is equal to the valuation $v_{x}$ itself. We define the divisor class map as the following morphism:

$$
d_{X}^{0}: K_{*}^{M}(E) \stackrel{\sum_{x} \partial_{v_{x}}}{\longrightarrow} \bigoplus_{x \in X^{(1)}} K_{*}^{M}(\kappa(x)) .
$$

This is an homogeneous morphism of degree -1 . According to what was said before, the reader can see that, in degree 1, it is precisely the usual divisor class map.

One can extend this formula to arbitrary algebraic $k$-schemes by using normalization of schemes and transfers in Milnor K-theory 2 The theory of cycle modules, or rather the intermediary notion of a cycle premodule, is an axiomatization of the functorial properties of Milnor K-theory, as a functor defined over function fields over $k$, which allows to use the same formula. In the end, one obtains for a cycle premodule $M$ over $k$, an algebraic $k$-scheme $X$ and an integer $n \geq 0$ a canonical morphism

$$
d_{X, M}^{n}: \bigoplus_{x \in X^{(n)}} M(\kappa(x)) \longrightarrow \prod_{y \in X^{(n+1)}} M(\kappa(y))
$$

homogeneous of degree -1 . Recall the first axiom of a cycle module says this map actually lands in the direct sum over $y \in X^{(n+1)}$.

The bridge between Rost's cycle modules and Voevodsky's motives was built in the thesis of the author. We defined two reciprocal equivalences of categories between cycle modules and certain graded sheaves obtained by a stabilization process from the homotopy invariant sheaves with transfers of the theory of Voevodsky (see Dég09, Th. 3.4]). This can be seen as an elaboration on the fact these latter sheaves admit a Gersten resolution 3 as proved by Voevodsky (FSV00, chap. 3, 4.37]). Of the results of our thesis, we will need only the following one:

Theorem (Dég08, th. 6.2.1). Let $k$ be a perfect field, $D M_{g m}(k)$ be the category of geometrical non effective motives of Voevodsky over $k$ and $\mathscr{A}$ be a Grothendieck abelian category.

Let $H: D M_{g m}(k)^{o p} \rightarrow \mathscr{A}$ be a cohomological functorn. Then for any couple $(q, n) \in \mathbb{Z}^{2}$, there exists a canonical cycle premodule $\hat{H}_{*}^{q, n}$ with coefficient 5 的 $\mathscr{A}$ such that for any integral $k$-scheme $X$ with function field $E$,

$$
\hat{H}_{r}^{q, n}(E)=\underset{U \subset X}{\lim _{U C X}} H(M(U)(n+r)[q+r])
$$

where the limit runs over non empty smooth open subschemes $U$ of $X, M(U)$ being the motive associated with $U$.

Given a functor $H$ as in the above theorem, one can apply the usual considerations alluded to above and get a coniveau spectral sequence. To describe it, we

\footnotetext{
${ }^{2}$ The divisor class map in Milnor K-theory was first written down by Kato in Kat86, for arbitrary excellent schemes.

${ }^{3}$ In other words, their restriction to any smooth $k$-scheme are Cohen-Macaulay in the sense of Har66 Def. p. 238].

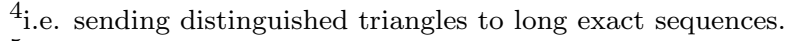

${ }^{5}$ Actually, Rost in Ros96] defines cycles modules and premodules only with coefficients in the category of abelian groups but the generalization to arbitrary abelian category is immediate.
} 
introduce the following useful notation: for any smooth $k$-scheme $X$ and any triple of integers $(q, n, r)$, we put:

$$
H_{r}^{q, n}(X):=H(M(X)(q+r, n+r)) .
$$

In our case, given a smooth $k$-scheme $X$ and an integer $n \in \mathbb{Z}$, the coniveau spectral sequence takes the form:

$$
E_{1}^{p, q}(X, n)=\bigoplus_{x \in X^{(p)}} \hat{H}_{*}^{q, n}(\kappa(x)) \Rightarrow H_{*}^{p+q, n}(X),
$$

with coefficients in the category of graded objects of $\mathscr{A}$, differentials in the $E_{1}$ page being homogeneous of degree -1 . It converges as required to the coniveau filtration on the cohomology $H$ - see (2.1.C) in the text. Explicitly, the $p$-th filtered part of this filtration is made of cohomology classes of $X$ which vanish on an open subscheme of $X$ with complement of codimension at least $p$ in $X$.

With these notations, we can now state the main result of this paper:

Proposition (Prop. 2.7). Consider a cohomological functor $H: D M_{g m}(k)^{o p} \rightarrow \mathscr{A}$ as above and denote by $d_{1}^{p, q}$ the differentials of the spectral sequence (函).

Then for any couple of integers $(p, q) \in \mathbb{Z}^{2}$,

$$
d_{1}^{p, q}=d_{\hat{H}^{q, n}}^{p} .
$$

This gives back the computation of Quillen but replacing K-theory with motivic cohomology - it can be shown this amounts to the same using the more involved work of [Dég10. The most interesting fact is that we obtain as a corollary that the divisor maps $d_{\hat{H}_{*}^{q, n}}^{*}$ induce a well defined complex: in the terminology of Rost, $\hat{H}_{*}^{q, n}$ is a cycle module. We already proved this fact in Dég08 using results of Rost and the fact the field $k$ is perfect. The proof given here is much more direct and geometric. To be precise, it relies on our work on the Gysin triangle Dég11 - the main ingredient is the functoriality of residues with respect to Gysin morphisms.

As apparent from the beginning, this study is closely connected with the pioneering work of Bloch and Ogus. In fact, as a corollary of the preceding computation together with the results of [Ros96] and Dég09, we get back the results of Bloch and Ogus for the cohomology $H$. More precisely:

Theorem (Prop. 2.12 and 2.14). Consider as above a cohomological functor $H$ : $D M_{g m}(k)^{o p} \rightarrow \mathscr{A}$. For any smooth scheme $X$, let us denote by $\mathcal{H}_{*}^{q, n}(X)$ the kernel of the divisor map $d_{X, \hat{H}_{*}^{q, n}}^{0}$.

Then $\mathcal{H}_{*}^{q, n}$ is a homotopy invariant Nisnevich sheaf with transfers in the sense of Voevodsky. It coincides with the Zariski sheaf associated with $H_{*}^{q, n}$.

The coniveau spectral sequence (荬) can be written from $E_{2}$ on as:

$$
E_{2}^{p, q}(X, n)=H_{\text {Zar }}^{p}\left(X, \mathcal{H}_{*}^{q, n}\right) \simeq H_{\mathrm{Nis}}^{p}\left(X, \mathcal{H}_{*}^{q, n}\right) \Rightarrow H_{*}^{p+q, n}(X) .
$$

Let us finish this introduction with a concrete application of these theoretical results. Let $k$ be a perfect field of characteristic $p>0, W$ its Witt ring and $K$ the fraction field of $W$. Given a smooth (resp. smooth affine) $k$-scheme $X$, we denote by $H_{c r y s}^{*}(X / W)$ (resp. $\left.H_{M W}^{*}(X)\right)$ the crystalline cohomology (resp. Monsky-Washnitzer) of $X$ defined in [Ber74] (resp. [MW68]).

Then the following properties hold:

- Let $\mathcal{H}_{M W}^{*}$ be the Zariski sheaf on the category of smooth $k$-schemes associated with the presheaf $H_{M W}^{*}$. 
Then $\mathcal{H}_{M W}^{*}$ is a homotopy invariant Nisnevich sheaf with transfers and $\Gamma\left(X, \mathcal{H}_{M W}^{*}\right)$ is invariant on the birational class of a smooth proper scheme $X$.

- For any smooth proper scheme $X$, there exists a spectral sequence

$$
E_{2}^{p, q}=H_{\text {Zar }}^{p}\left(X, \mathcal{H}_{M W}^{q}\right) \Rightarrow H_{\text {crys }}^{p+q}(X / W) \otimes K
$$

converging to the coniveau filtration on $H_{c r y s}^{i}(X / W)_{K}$.

- When $k$ is separably closed, for any $p \geq 0$,

$$
H_{\text {Zar }}^{p}\left(X, \mathcal{H}_{M W}^{p}\right)=A^{p}(X) \otimes K
$$

where the right hand side is the group of $p$-codimensional cycles modulo algebraic equivalence.

This set of properties is a corollary of the preceding theorem when one uses the rigid cohomology defined by Berthelot (e.g. [Ber97]) together with its realization $H_{r i g}: D M_{g m}(k)^{o p} \rightarrow K-v s$ introduced in the work of Cisinski and the author on Mixed Weil cohomologies [CD07] - see Remark 2.16 for details.

Organization of the paper. Section 1 is the technical heart of the paper. We introduce the new notion of a triangulated exact couple (Def. 1.1), associated with any filtered object of a triangulated category together with a choice of cones. Our main example is what we call the motivic coniveau exact couple (Def. 1.6) which, through a realization functor of mixed motives, universally gives rise to the exact couple corresponding to the coniveau spectral sequence. In section 1.2 we give the computation of the differentials of this exact couple in terms of generic motives (recalled in section 1.2.1). Section 2 relates this computation with the theory of cycle modules through cohomological realizations and gives a proof of the results stated in this introduction.

Foreword. There has been a lot of work on the coniveau spectral sequence apart those cited in the introduction. Let us mention in particular [CTHK97] which gives, using a proof of Gabber, the computation of the $E_{2}$-term as in the above theorem in a more general setting - which does not require homotopy invariance. It can be applied to the example given above by using in particular the étale descent theorem of [CT03 for rigid cohomology - this is well known to imply Nisnevich excision as required in CTHK97.

Our contribution to the story is made of the following points:

- The unramified cohomology sheaves $\mathcal{H}^{q, n}$, in our setting, admits a canonical structure of a homotopy invariant sheaf with transfers - a particular case of motivic complex.

- The differentials in the $E_{1}$-term can be computed in terms of Rost theory through an explicit cycle module.

- We have extended the identification of the diagonal terms in the $E_{2}$-page of the spectral sequence to cycles modulo algebraic equivalence, proved in characteristic 0 in BO74, to the case of characteristic $p$ for a reasonable cohomology theory (see the example above and Corollary 2.25 for the general case).

\section{NOTATIONS AND CONVENTIONS}

We fix a base field $k$ which is assumed to be perfect. The word scheme will stand for any separated $k$-scheme of finite type, and we will say that a scheme is smooth 
when it is smooth over the base field. The category of smooth schemes is denoted by $\mathscr{S} m_{k}$.

We will also use the theory of geometric motives defined by Voevodsky in FSV00, chap. 5]). Therefore, we denote by $\mathscr{S} m_{k}^{\text {cor }}$ the category of smooth schemes with morphisms the finite correspondences. This is an additive category whose objects are denoted under bracket - eg $[X]$. The category of geometric effective motives, denoted by $D M_{g m}^{e f f}(k)$, is a quotient of the bounded homotopy category associated with $\mathscr{S} m_{k}^{\text {cor }}$. The motive $M(X)$ of a smooth scheme $X$ is the complex equal to $[X]$ placed in degree 0 . Given an open subscheme $U$ of a smooth scheme $X$, we will define the relative motive of $X$ modulo $U$ as the cone of the canonical immersion $U \rightarrow X$ computed in the category of complexes with coefficients in $\mathscr{S} m_{k}^{c o r}$ :

$$
M(X / U):=\operatorname{Cone}([U] \rightarrow[X]) .
$$

This motive is functorial with respect to morphisms of schemes which stabilize the open subscheme.

The category $D M_{g m}^{e f f}(k)$ is monoidal, with unit $\mathbb{Z}:=M(\operatorname{Spec}(k))$. Recall the Tate twist can be defined as:

$$
\mathbb{Z}(1):=M\left(\mathbb{A}_{k}^{1} / \mathbb{G}_{m}\right)[-2] .
$$

The category of geometric motives $D M_{g m}(k)$ is the stabilization of $D M_{g m}^{e f f}(k)$ with respect to $\mathbb{Z}(1)$ - i.e. the monoidal category obtained by formally inverting this object with respect to the tensor product. There is a canonical functor

$$
\Sigma^{\infty}: D M_{g m}^{e f f}(k) \rightarrow D M_{g m}(k)
$$

and we will still denote by $M(X)$ (resp. $M(X / U)$ ) the image of $M(X)$ (resp. $M(X / U))$ by $\Sigma^{\infty}$.

According to the theory of Voevodsky, a homotopy invariant sheaf with transfers with values in an abelian category $\mathscr{A}$ will be a functor $F:\left(\mathscr{S} m_{k}^{\text {cor }}\right)^{o p} \rightarrow \mathscr{A}$ which is a sheaf for the Nisnevich topology and such that for any smooth scheme $X$, the map $F(X) \rightarrow F\left(\mathbb{A}_{X}^{1}\right)$ induced by the projection is an isomorphism.

We will use the following constructions of Dég11:

- Given a pair $(X, Z)$ such that $X$ (resp. $Z$ ) is a smooth scheme (resp. smooth closed subscheme of $X$ of pure codimension $n$ ), we defined a purity isomorphism in [Dég11, Prop. 1.12]:

(Intro.a) $\quad \mathfrak{p}_{(X, Z)}: M(X / X-Z) \rightarrow M(Z)(n)[2 n]$.

- Given a projective morphism $f: Y \rightarrow X$ between smooth schemes of pure dimension $d$, we defined a Gysin morphism in Dég11, Def. 2.7]:

(Intro.b)

$$
f^{*}: M(X)(d)[2 d] \rightarrow M(Y) \text {. }
$$

\section{Contents}

Introduction

Notations and conventions

1. Motivic coniveau exact couple

1.1. Definition

1.2. Computations

. Cohomological realization

2.1. The coniveau spectral sequence

2.2. Cycle modules

2.3. Algebraic equivalence 20 


\section{Motivic Coniveau exact Couple}

\subsection{Definition.}

1.1.1. Triangulated exact couple. We introduce a triangulated version of the classical exact couples.

Definition 1.1. Let $\mathscr{T}$ be a triangulated category. A triangulated exact couple is the data of bigraded objects $D$ and $E$ of $\mathscr{T}$ and homogeneous morphisms between them

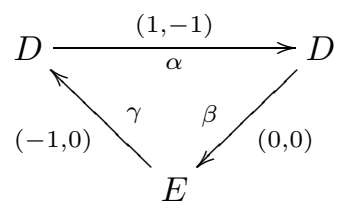

with the bidegrees of each morphism indicated in the diagram and such that the above triangle is a distinguished triangle in each bidegree 6

Given such a triangulated exact couple, we will usually put $d=\beta \circ \gamma$, homogeneous endomorphism of $E$ of bidegree $(-1,0)$. We easily get that $d^{2}=0$, thus obtaining a complex

$$
\ldots \rightarrow E_{p, q} \stackrel{d_{p, q}}{\longrightarrow} E_{p-1, q} \rightarrow \ldots
$$

Let $\mathscr{A}$ be an abelian category. A cohomological functor with values in $\mathscr{A}$ is an additive functor $H: \mathscr{T}^{o p} \rightarrow \mathscr{A}$ which sends distinguished triangles to long exact sequences. For $p$ an integer, we simply put $H^{p}=H \circ .[-p]$.

Apply the contravariant functor $H=H^{0}$ to the diagram (1.1.a), we naturally obtain a commutative diagram of bigraded objects of $\mathscr{A}$ :

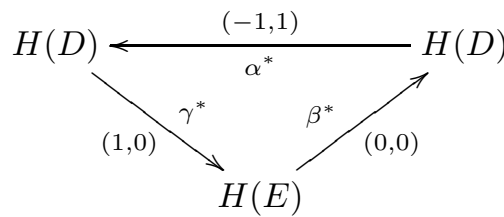

This is an exact couple of $\mathscr{A}$ in the classical sense (following the convention of [McC01, th. 2.8]). Thus we can associate with this exact couple a spectral sequence:

$$
E_{1}^{p, q}=H\left(E_{p, q}\right)
$$

with differentials being $H\left(d_{p, q}\right): H\left(E_{p-1, q}\right) \rightarrow H\left(E_{p, q}\right)$.

Definition 1.2. Let $\mathscr{T}$ be a triangulated category and $X$ an object of $\mathscr{T}$.

(1) A tower $X_{\bullet}$ over $X$ is the data of a sequence $\left(X_{p} \rightarrow X\right)_{p \in \mathbb{Z}}$ of objects over $X$ and a sequence of morphisms over $X$

$$
. . \rightarrow X_{p-1} \stackrel{j_{p}}{\rightarrow} X_{p} \rightarrow \ldots
$$

\footnotetext{
${ }^{6}$ Note this implies in particular the relation $D_{p, q+1}=D_{p, q}[-1]$ for any couple of integers $(p, q)$.
} 
(2) Let $X$ • be a tower over $X$. Suppose that for each integer $p$ we are given a distinguished triangle

$$
X_{p-1} \stackrel{j_{p}}{\longrightarrow} X_{p} \stackrel{\pi_{p}}{\longrightarrow} C_{p} \stackrel{\delta_{p}}{\longrightarrow} X_{p}[1]
$$

where $j_{p}$ is the structural morphism of the tower $X_{\bullet}$.

Then we associate with the tower $X_{\bullet}$ and the choice of cones $C_{\bullet}$ a triangulated exact couple

$$
D_{p, q}=X_{p}[-p-q], \quad E_{p, q}=C_{p}[-p-q]
$$

with structural morphisms

$$
\alpha_{p, q}=j_{p}[-p-q], \beta_{p, q}=\pi_{p}[-p-q], \gamma_{p, q}=\delta_{p}[-p-q] .
$$

Let $H: \mathscr{T}^{o p} \rightarrow \mathscr{A}$ be a cohomological functor. In the situation of this definition, we thus have a spectral sequence of $E_{1}$-term: $E_{1}^{p, q}=H^{p+q}\left(C_{p}\right)$.

We consider the case where $X \bullet$ is bounded and exhaustive i.e.

$$
X_{p}= \begin{cases}0 & \text { if } p \ll 0 \\ X & \text { if } p \gg 0 .\end{cases}
$$

In this case, the spectral sequence is concentrated in a band with respect to $p$ and we get a convergent spectral sequence

$$
E_{1}^{p, q}=H^{p+q}\left(C_{p}\right) \Rightarrow H^{p+q}(X) .
$$

The filtration on the abutment is then given by the formula

$$
F_{i l t}^{r}\left(H^{p+q}(X)\right)=\operatorname{Ker}\left(H^{p+q}(X) \rightarrow H^{p+q}\left(X_{r}\right)\right) .
$$

1.1.2. Definition. In the next definitions, we introduce our main example of an exact couple, based on a filtration by certain open subsets.

Definition 1.3. Let $X$ be a scheme.

A flag on $X$ is a decreasing sequence $\left(Z^{p}\right)_{p \in \mathbb{N}}$ of closed subschemes of $X$ such that for all integer $p \geq 0, Z^{p}$ is of codimension greater or equal to $p$ in $X$. We let $\mathcal{D}(X)$ be the set of flags of $X$, ordered by termwise inclusion.

We will consider a flag $\left(Z^{p}\right)_{p \in \mathbb{N}}$ has a $\mathbb{Z}$-sequence by putting $Z^{p}=X$ for $p<0$. It is an easy fact that, with the above definition, $\mathcal{D}(X)$ is right filtering.

Recall that a pro-object of a category $\mathcal{C}$ is a (covariant) functor $F$ from a left filtering category $\mathcal{I}$ to the category $\mathcal{C}$. Usually, we will denote $F$ by the intuitive notation "lim $\varliminf_{i \in \mathcal{I}} F_{i}$ and call it the formal projective limit.

Definition 1.4. Let $X$ be a scheme. We define the coniveau filtration of $X$ as the sequence $\left(F_{p} X\right)_{p \in \mathbb{Z}}$ of pro-open subschemes of $X$ such that :

$$
F_{p} X=Z_{Z^{*} \in \varliminf_{\mathcal{D}(X)}{ }^{o p}}\left(X-Z^{p}\right)
$$

We denote by $j_{p}: F_{p-1} X \rightarrow F_{p} X$ the canonical pro-open immersion,

$$
j_{p}={ }_{Z^{*} \in \overleftarrow{\mathcal{D}(X)^{o p}}}\left(\left(X-Z^{p-1}\right) \rightarrow\left(X-Z^{p}\right)\right) .
$$

Remark 1.5. Usually, the coniveau filtration occurs on cohomology. As we will see below, the filtration we have just introduced on a scheme will give rise to the classical coniveau filtration, on cohomology. Therefore, we think our terminology is both handy and sufficiently accurate. 
Unfortunately, this is a filtration by pro-schemes, and if we apply to it the functor $M$ termwise, we obtain a filtration of $M(X)$ in the category pro- $D M_{g m}^{e f f}(k)$. This latter category is never triangulated. Nonetheless, the definition of an exact couple still makes sense for the pro-objects of a triangulated category if we replace distinguished triangles by pro-distinguished triangles]. We consider the tower of pro-motives above the constant pro-motive $M(X)$

$$
\ldots \rightarrow M\left(F_{p-1} X\right) \stackrel{j_{p *}}{\longrightarrow} M\left(F_{p} X\right) \rightarrow \ldots
$$

We define the following canonical pro-cone

$$
G r_{p}^{M}(X)=\underset{Z^{*} \in{\overleftarrow{\mathcal{D}}(X)^{o p}}^{o p}}{\lim } M\left(X-Z^{p} / X-Z^{p-1}\right)
$$

using relative motives - see the general notations of the article. We thus obtain pro-distinguished triangles:

$$
M\left(F_{p-1} X\right) \stackrel{j_{p *}}{\longrightarrow} M\left(F_{p} X\right) \stackrel{\pi_{p}}{\longrightarrow} G r_{p}^{M}(X) \stackrel{\delta_{p}}{\longrightarrow} M\left(F_{p-1} X\right)[1] .
$$

Definition 1.6. Consider the above notations. We define the motivic coniveau exact couple associated with $X$ in pro- $D M_{g m}^{e f f}(k)$ as

$$
D_{p, q}=M\left(F_{p} X\right)[-p-q], \quad E_{p, q}=G r_{p}^{M}(X)[-p-q],
$$

with structural morphisms

$$
\alpha_{p, q}=j_{p}[-p-q], \beta_{p, q}=\pi_{p}[-p-q], \gamma_{p, q}=\delta_{p}[-p-q] .
$$

According to the notation which follows Definition 1.1, the differential associated with the motivic coniveau exact couple is equal to the composite map of the following diagram:

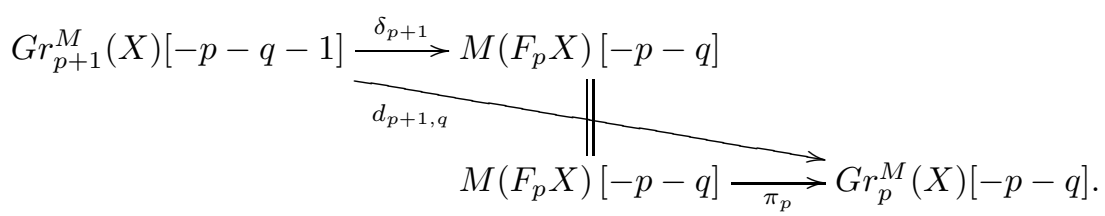

\subsection{Computations.}

\subsubsection{Recollection and complement on generic motives.}

1.7. We will call function field any finite type field extension $E / k$. A model of the function field $E$ will be a connected smooth scheme $X / k$ with a given $k$-isomorphism between the function field of $X$ and $E$. Recall the following definition from Dég08, 3.3.1] :

Definition 1.8. Consider a function field $E / k$ and an integer $n \in \mathbb{Z}$. We define the generic motive of $E$ with weight $n$ as the following pro-object of $D M_{g m}(k)$ :

$$
M(E)(n)[n]:={ }_{A \subset E, \operatorname{Spec}(\overleftarrow{A}) \text { model of } E / k} M(\operatorname{Spec}(A))(n)[n]
$$

We denote by $D M_{g m}^{(0)}(k)$ the full subcategory of pro- $D M_{g m}(k)$ consisting of the generic motives.

Of course, given a function field $E$ with model $X / k$, the pro-object $M(E)$ is canonically isomorphic to the pro-motive made by the motives of non empty open subschemes of $X$.

7 i.e. the formal projective limit of distinguished triangles. 
1.9. The interest of generic motives lies in their functoriality which we now review : (1) Given any extension of function fields $\varphi: E \rightarrow L$, we get a morphism $\varphi^{*}$ : $M(L) \rightarrow M(E)$ (by covariant functoriality of motives).

(2) Consider a finite extension of function fields $\varphi: E \rightarrow L$. One can find respective models $X$ and $Y$ of $E$ and $L$ together with a finite morphism of schemes $f$ : $Y \rightarrow X$ which induces on function fields the morphism $\varphi$ through the structural isomorphisms.

For any open subscheme $U \subset X$, we put $Y_{U}=Y \times_{X} U$ and let $f_{U}: Y_{U} \rightarrow U$ be the morphism induced by $f$. It is finite and surjective. In particular, its graph seen as a cycle in $U \times Y_{U}$ defines a finite correspondence from $U$ to $Y_{U}$, denoted by ${ }^{t} f_{U}$ and called the transpose of $f_{U}$. We define the norm morphism $\varphi_{*}: M(E) \rightarrow M(L)$ as the well defined pro-morphism (see [Dég08, 5.2.9])

$$
" \varliminf_{U \subset X} "\left(M(U) \stackrel{\left(\left.{ }^{t} f\right|_{U}\right)_{*}}{\longrightarrow} M\left(Y_{U}\right)\right)
$$

through the structural isomorphisms of the models $X$ and $Y$.

(3) Consider a function field $E$ and a unit $x \in E^{\times}$. Given a smooth sub- $k$-algebra $A \subset E$ which contains $x$ and $x^{-1}$, we get a morphism $\operatorname{Spec}(A) \rightarrow \mathbb{G}_{m}$. Let us denote by $\gamma_{x}^{A}: \operatorname{Spec}(A) \rightarrow \operatorname{Spec}(A) \times \mathbb{G}_{m}$ its graph. Recall the canonical decomposition $M\left(\mathbb{G}_{m}\right)=\mathbb{Z} \oplus \mathbb{Z}(1)[1]$ and consider the associated projection $M\left(\mathbb{G}_{m}\right) \stackrel{\pi}{\rightarrow} \mathbb{Z}(1)[1]$. We associate with the unit $x$ a morphism $\gamma_{x}: M(E) \rightarrow M(E)(1)[1]$ defined as follows:

$$
\underset{x, x^{-1} \in A \subset E}{" \lim }\left(M(\operatorname{Spec}(A)) \stackrel{\gamma_{x *}^{A}}{\longrightarrow} M(\operatorname{Spec}(A)) \otimes M\left(\mathbb{G}_{m}\right) \stackrel{\pi}{\rightarrow} M(\operatorname{Spec}(A))(1)[1]\right) .
$$

One can prove moreover that if $x \neq 1, \gamma_{x} \circ \gamma_{1-x}=0$ and $\gamma_{1-x} \circ \gamma_{x}=0$ so that any element $\sigma \in K_{n}^{M}(E)$ of Milnor K-theory defines a morphism $\gamma_{\sigma}: M(E) \rightarrow$ $M(E)(n)[n]$ (see Dég08, 5.3.5]).

(4) Let $E$ be a function field and $v$ a discrete valuation on $E$ with ring of integers $\mathcal{O}_{v}$ essentially of finite type over $k$. Let $\kappa(v)$ be the residue field of $v$.

As $k$ is perfect, there exists a connected smooth scheme $X$ with a point $x \in X$ of codimension 1 such that $\mathcal{O}_{X, x}$ is isomorphic to $\mathcal{O}_{v}$. This implies $X$ is a model of $E / k$. Moreover, reducing $X$, one can assume the closure $Z$ of $x$ in $X$ is smooth so that it becomes a model of $\kappa(v)$.

For an open neighborhood $U$ of $x$ in $X$, we put $Z_{U}=Z \times{ }_{X} U$. We define the residue morphism $\partial_{v}: M(\kappa(v))(1)[1] \rightarrow M(E)$ associated with $(E, v)$ as the pro-morphism

$$
\text { "lim } \lim _{x \in U \subset X}\left(M\left(Z_{U}\right)(1)[1] \stackrel{\partial_{U}, Z_{U}}{\longrightarrow} M\left(U-Z_{U}\right)\right) .
$$

The fact it is a morphism of pro-objects follows from the functoriality of residues with respect to open immersions (see [Dég08, 5.4.6]).

Remark 1.10. These morphisms satisfy a set of relations which in fact corresponds exactly to the axioms of a cycle premodule by M. Rost (cf [Ros96, (1.1)]). We refer the reader to [Dég08, 5.1.1] for a precise statement.

1.11. Consider again the situation and notations of point (2) in paragraph 1.9 With the Gysin morphism we have introduced before, one can give another definition for the norm morphism of generic motives.

Indeed, for any open subscheme $U$ of $X$, the morphism $f_{U}: Y_{U} \rightarrow U$ is finite of relative dimension 0 and thus induces a Gysin morphism $f_{U}^{*}: M(U) \rightarrow M\left(Y_{U}\right)-$ 
see (Intro.b). The morphism $f_{U}^{*}$ is natural with respect open immersions according to Dég11, Prop. 2.10]. Thus, we get a morphism of pro-objects

$$
\left." \varliminf_{U \subset X} "\left(M(U) \stackrel{f_{U}^{*}}{\longrightarrow} M\left(Y_{U}\right)\right)\right) .
$$

which induces through the structural isomorphisms of the models $X$ and $Y$ a morphism $\varphi_{*}^{\prime}: M(E) \rightarrow M(L)$.

Lemma 1.12. Consider the above notations. Then, $\varphi_{*}^{\prime}=\varphi_{*}$.

Proof. By functoriality, we can restrict the proof to the cases where $L / E$ is separable or $L / E$ is purely inseparable.

In the first case, we can choose a model $f: Y \rightarrow X$ of $\varphi$ which is étale. Then the lemma follows from Dég11, Prop. 2.15].

In the second case, we can assume that $L=E[\sqrt[a]{a}]$ for $a \in E$. Let $A \subset E$ be a sub-k-algebra containing $a$ such that $X=\operatorname{Spec}(A)$ is a smooth scheme. Let $B=A[t] /\left(t^{q}-a\right)$. Then $Y=\operatorname{Spec}(B)$ is again a smooth scheme (over $k$ ) and the canonical morphism $f: Y \rightarrow X$ is a model of $L / E$. We consider its canonical factorisation $Y \stackrel{i}{\rightarrow} \mathbb{P}_{X}^{1} \stackrel{p}{\rightarrow} X$ corresponding to the parameter $t$, together with the following diagram made of two cartesian squares:

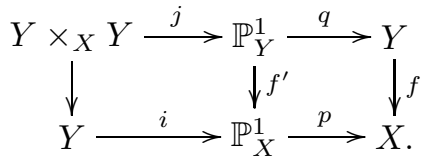

The scheme $Y \times_{X} Y$ is non reduced and its reduction is $Y$. Moreover, the canonical immersion $Y \rightarrow Y \times_{X} Y$ is an exact thickening of order $q$ in $Y$ according to the definition of [Dég11, Par. 1.18]. Thus, the following diagram is commutative :

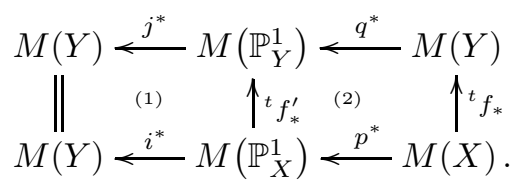

Indeed, part (2) (resp. (1)) is commutative by Dég08, 2.2.15] (resp. Dég08, 2.5.2: (2)]). Thus $f^{*}={ }^{t} f_{*}$ and this concludes.

1.2.2. The graded terms. For a scheme $X$, we denote by $X^{(p)}$ the set of points of $X$ of codimension $p$. If $x$ is a point of $X, \kappa(x)$ will denote its residue field. The symbol " $\prod "$ denotes the product in the category of pro-motives.

Lemma 1.13. Let $X$ be a smooth scheme and consider the notations of Definition 1.6. Then, for all integer $p \geq 0$, purity isomorphisms of the form (Intro.a induce a canonical isomorphism

$$
G r_{p}^{M}(X) \stackrel{\epsilon_{p}}{\longrightarrow} " \prod_{x \in X^{(p)}} " M(\kappa(x))(p)[2 p] .
$$

In particular, for any point $x \in X^{(p)}$ we get a canonical projection map:

$$
\pi_{x}: G r_{p}^{M}(X) \rightarrow M(\kappa(x))(p)[2 p] .
$$


Proof. Let $\mathcal{I}_{p}$ be the set of pairs $\left(Z, Z^{\prime}\right)$ such that $Z$ is a reduced closed subscheme of $X$ of codimension $p$ and $Z^{\prime}$ is a closed subset of $Z$ containing its singular locus. Then

$$
G r_{p}^{M}(X) \simeq \underset{\left(Z, \overleftarrow{\left.Z^{\prime}\right) \in \mathcal{I}_{p}}\right.}{" \lim "} M\left(X-Z^{\prime} / X-Z\right)
$$

For any element $\left(Z, Z^{\prime}\right)$ of $\mathcal{I}_{p}$, under the purity isomorphism (Intro.a), we get: $M\left(X-Z^{\prime} / X-Z\right) \simeq M\left(Z-Z^{\prime}\right)(p)[2 p]$.

For any point $x$ of $X$, we let $Z(x)$ be the reduced closure of $x$ in $X$ and $\mathcal{F}(x)$ be the set of closed subschemes $Z^{\prime}$ of $Z(x)$ containing the singular locus $Z(x)_{\operatorname{sing}}$ of $Z(x)$. By additivity of motives, we finally get an isomorphism:

$$
G r_{p}^{M}(X) \simeq " \prod_{x \in X^{(p)}} " Z_{Z^{\prime} \in \mathcal{F}(x)} M\left(Z(x)-Z^{\prime}\right)(p)[2 p] .
$$

This implies the lemma because $Z(x)-Z(x)_{\text {sing }}$ is a model of $\kappa(x)$.

\subsubsection{The differentials.}

1.14. Let $X$ be a scheme essentially of finite typ 8 over $k$ and consider a couple $(x, y) \in X^{(p)} \times X^{(p+1)}$.

Assume that $y$ is a specialisation of $x$. Let $Z$ be the reduced closure of $x$ in $X$ and $\tilde{Z} \stackrel{f}{\rightarrow} Z$ be its normalisation. Each point $t \in f^{-1}(y)$ corresponds to a discrete valuation $v_{t}$ on $\kappa(x)$ with residue field $\kappa(t)$. We denote by $\varphi_{t}: \kappa(y) \rightarrow \kappa(t)$ the morphism induced by $f$. Then, we define the following morphism of generic motives

$$
\partial_{y}^{x}=\sum_{t \in f^{-1}(y)} \partial_{v_{t}} \circ \varphi_{t *}: M(\kappa(y))(1)[1] \rightarrow M(\kappa(x))
$$

using the notations of 1.9

If $y$ is not a specialisation of $x$, we put conventionally $\partial_{y}^{x}=0$.

Proposition 1.15. Consider the above hypothesis and notations. If $X$ is smooth then the following diagram is commutative:

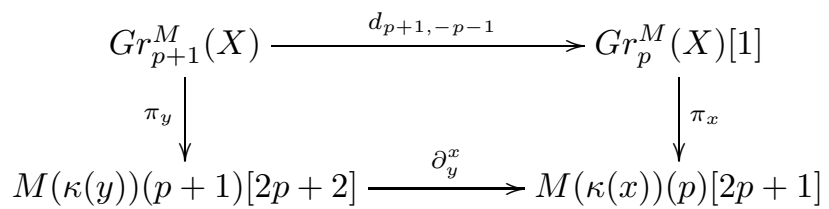

where the vertical maps are defined in (1.13.a) and $d_{p+1,-p-1}$ in (1.6.a).

Of course, this proposition determines every differentials of the motivic coniveau exact couple as $d_{p, q}=d_{p,-p}[-p-q]$.

Proof. According to Definition 1.6, the morphism $d_{p+1,-p-1}$ is the formal projective limit of the morphisms

$$
M(X-W / X-Y) \rightarrow M(X-Y)[1] \rightarrow M(X-Y / X-Z)[1],
$$

for large enough closed subsets $W \subset Y \subset Z$ of $X$ such that $\operatorname{codim}_{X}(Z)=p$, $\operatorname{codim}_{X}(Y)=p+1$ and $\operatorname{codim}_{X}(W) \geq p+2$. For the proof, we will consider $W \subset Y \subset Z$ as above, assume that $y \in Y, x \in Z$ and study (1.15.a) for $Z, Y, W$ large enough. To simplify the notations, we will replace $X$ by $X-W$ which means

\footnotetext{
${ }^{8}$ For the purpose of the next proposition, we need only the case where $X$ is smooth but the general case treated here will be used later.
} 
practically that we can substract any subset of $X$ if it has codimension greater than $p+1$.

First of all, enlarging $Y$, we can assume that it contains the singular locus of $Z$. Because the singular locus of $Y$ has codimension greater than $p+1$ in $X$, we can assume by reducing $X$ that $Y$ is smooth. Then, using the purity isomorphism, the composite map (1.15.a) is isomorphic to the following one:

$$
M(Y)((p+1)) \stackrel{\partial_{X, Y}}{\longrightarrow} M(X-Y)[1] \stackrel{i_{Y}^{*}}{\longrightarrow} M(Z-Y)((p))[1]
$$

where $i_{Y}:(Z-Y) \rightarrow(X-Y)$ is the obvious restriction of the canonical closed immersion $i: Y \rightarrow Z$.

Let $Y_{y}$ (resp. $Z_{x}$ ) be the irreducible component of $Y$ (resp. $Z$ ) containing $y$ (resp. $x$ ). As $Y$ is smooth, we can write $Y=Y_{y} \sqcup Y_{y}^{\prime}$ where $Y_{y}^{\prime}$ is the complement of $Y_{y}$ in $Y$. As $(Z-Y)$ is smooth, if we put $\hat{Y}_{x}=Y \times_{Z} Z_{x}$ then $\left(Z_{x}-\hat{Y}_{x}\right)$ is a connected component of $(Z-Y)$. We denote by $i_{x}:\left(Z_{x}-\hat{Y}_{x}\right) \rightarrow(X-Y)$ the obvious restriction of $i_{Y}$. According to [Dég11, Prop. 1.36], the following diagram is commutative:

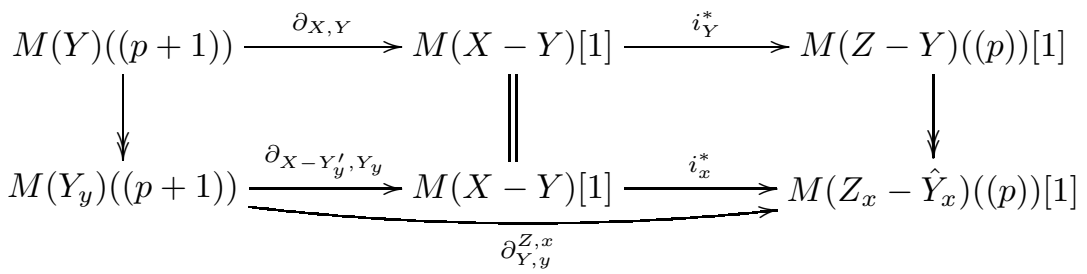

where the vertical maps are the canonical projections. The proposition is equivalent to show that the formal projective limit of the maps $\partial_{Y, y}^{Z, x}$ for $Z, Y, W$ large enough is equal to $\partial_{y}^{x}$ (remember we have identified $X$ with $X-W$ ).

Assume that $y$ is not a specialisation of $x$. Then $Y_{y} \cap Z_{x}$ has codimension greater than $p+1$ in $X$. Therefore, reducing $X$ again, we can assume $Y_{y} \cap Z_{x}=\emptyset$. A fortiori, $Y_{y} \cap\left(Z_{x}-\hat{Y}_{x}\right)=\emptyset$ and we get the following cartesian square of closed immersions:

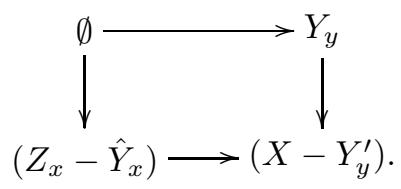

Then, according to the naturality of residues with respect to the preceding square

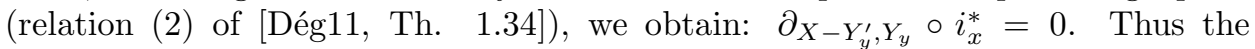
proposition is proved in that case.

We now consider the case where $y$ is a specialisation of $x$ i.e. $Y_{y} \subset Z_{x}$. Then $Y_{y} \subset \hat{Y}_{x}$ : to simplify the notation, we can assume that $Z=Z_{x}$ i.e. $Z$ is irreducible with generic point $x$. Let $f: \tilde{Z} \rightarrow Z$ be the normalization of $Z$. The singular locus $\tilde{Z}_{\text {sing }}$ of $\tilde{Z}$ is everywhere of codimension greater than 1 in $\tilde{Z}$. Thus, $f\left(\tilde{Z}_{\text {sing }}\right)$ is everywhere of codimension greater than $p+1$ in $X$, and we can assume by reducing $X$ again that $\tilde{Z}$ is smooth.

Let us denote by $\tilde{Y}$ (resp. $\tilde{Y}_{y}, \tilde{Y}_{y}^{\prime}$ ) the reduced inverse image of $Y$ (resp. $Y_{y}, Y_{y}^{\prime}$ ) along $f$. Reducing $X$ again, we can assume that $\tilde{Y}_{y}$ is smooth and $\tilde{Y}_{y} \cap \tilde{Y}_{y}^{\prime}=\emptyset$. Moreover, we can assume that every connected component of $\tilde{Y}_{y}$ dominates $Y_{y}$ (by reducing $X$, we can throw away the non dominant connected components). In other 
words, the map $g_{y}: \tilde{Y}_{y} \rightarrow Y_{y}$ induced by $f$ is finite and equidimensional. Then we can consider the following topologically cartesian square:

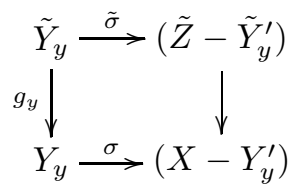

where $\sigma$ and $\tilde{\sigma}$ are the obvious closed immersions and the right vertical map is induced by the composite map $\tilde{Z} \stackrel{f}{\rightarrow} Z \stackrel{i}{\rightarrow} X$. Note that taking the respective complements of $\tilde{\sigma}$ and $\sigma$ in the source and target of this composite map, it induces the following one:

$$
(\tilde{Z}-\tilde{Y}) \stackrel{h}{\rightarrow}(Z-Y) \stackrel{i}{\rightarrow}(X-Y)
$$

Thus, applying the naturality of residues with respect to Gysin morphisms (Dég11, Prop. 2.13]) to the preceding square on the one hand and the functoriality of the Gysin morphism ([Dég11, Prop. 2.9]) on the other hand, we obtain the following commutative diagram:

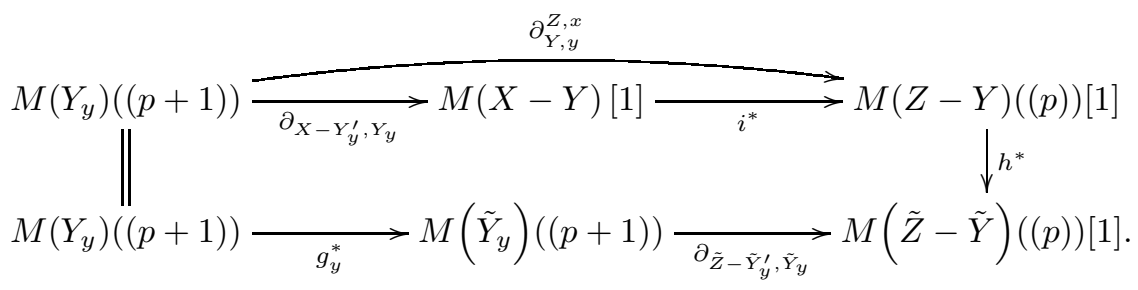

Note that the set of connected components of the smooth scheme $\tilde{Y}_{y}$ corresponds bijectively to the set $f^{-1}(y)$. For any $t \in f^{-1}(y)$, we denote by $\tilde{Y}_{t}$ the corresponding connected component so that $\tilde{Y}_{y}=\sqcup_{t \in f^{-1}(y)} \tilde{Y}_{t}$. Note that $\tilde{Y}_{t}$ is also a connected component of $\tilde{Y}$. We put:

$$
\tilde{Z}_{t}=\tilde{Z}-\left(\tilde{Y}-\tilde{Y}_{t}\right)
$$

This is an open subscheme of $\tilde{Z}$ containing $\tilde{Y}_{t}$ and $\left(\tilde{Z}_{t}-\tilde{Y}_{t}\right)=(\tilde{Z}-\tilde{Y})$. Finally applying the additivity properties of Gysin morphisms and residues ([Dég11, Prop. $1.36]$ ), we obtain the following commutative squares:

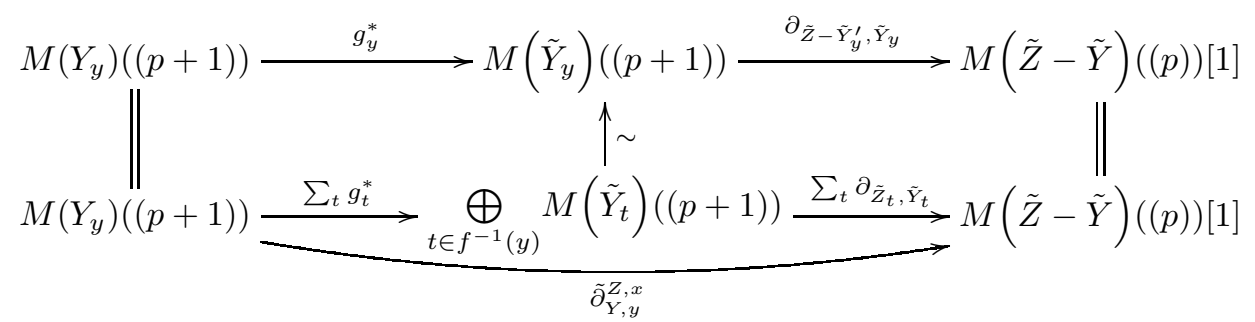

where the middle vertical map is the canonical isomorphism. We can now identify $\partial_{y}^{x}$ with the formal projective limit of $\tilde{\partial}_{Y, y}^{Z, x}$ for $Y, W$ large enough (remember we have assumed $Z=Z_{x}$ ). In view of formula (1.14.a), this is justified because:

- $h$ is birational and $(\tilde{Z}-\tilde{Y})$ is a smooth model of $\kappa(x)$.

- The closed pair $\left(\tilde{Z}_{t}, \tilde{Y}_{t}\right)$ is smooth of codimension 1 and the local ring of $\mathcal{O}_{\tilde{Z}_{t}, \tilde{Y}_{t}}$ is isomorphic (through $h$ ) to the valuation ring $\mathcal{O}_{v_{t}}$ corresponding to the valuation $v_{t}$ on $\kappa(x)$ considered in Paragraph 1.14 . 


\section{Cohomological ReAlization}

We fix a Grothendieck abelian category $\mathscr{A}$ and consider a cohomological functor

$$
H: D M_{g m}(k)^{o p} \rightarrow \mathscr{A},
$$

simply called a realization functor. To such a functor, we associate a twisted cohomology theory: for a smooth scheme $X$ and any pair of integers $(i, n) \in \mathbb{Z}^{2}$, we put:

$$
H^{i, n}(X)=H(M(X)(-n)[-i]) .
$$

By the very definition, this functor is contravariant, not only with respect to morphisms of smooth schemes but also for finite correspondences. Using Gysin morphisms (Intro.b), it is also covariant with respect to projective morphisms.

\subsection{The coniveau spectral sequence.}

2.1. The functor $H$ admits an obvious extension to pro-objects

$$
\bar{H}: \operatorname{pro}-D M_{g m}(k)^{o p} \rightarrow \mathscr{A}
$$

which sends pro-distinguished triangles to long exact sequences since right filtering colimits are exact in $\mathscr{A}$. Moreover, for any function fields $E / k$, we simply put

$$
\hat{H}^{i, n}(E):=\lim _{\overrightarrow{A \subset E}} H^{i, n}(\operatorname{Spec}(A))
$$

where the limit is taken over the models of the function field $E / k$ (see Paragraph 1.7).

Fix an integer $n \in \mathbb{Z}$. We apply the functor $\bar{H}(?(n))$ to the pro-exact couple of Definition 1.6. We then obtain a converging spectral sequence which, according to Lemma 1.13, has the form:

$$
E_{1}^{p, q}(X, n)=\bigoplus_{x \in X^{(p)}} \hat{H}^{q-p, n-p}(\kappa(x)) \Rightarrow H^{p+q, n}(X) .
$$

This is a spectral sequence which converges to the so-called coniveau filtration on the twisted cohomology $H^{* *}$ defined by:

$$
N^{r} H^{i, n}(X)=\bigcap_{U \subset X, \operatorname{codim}_{X}(X-U) \geq r} \operatorname{Ker}\left(H^{i, n}(X) \rightarrow H^{i, n}(U)\right) .
$$

We also call the above spectral sequence the coniveau spectral sequence of $X$ with coefficients in $H$.

2.2. Cycle modules. We now recall below the theory of Rost cycle modules in a way adapted to our needs.

2.2. The first step in Rost's theory is the notion of a cycle premodule. As already mentioned in the introduction, it is a covariant functor from the category of function fields to the category of graded abelian groups satisfying an enriched functoriality exactly analog to that of Milnor K-theory $K_{*}^{M}$. In our context, we will define9 a cycle premodule as a functor

$$
\phi: D M_{g m}^{(0)}(k)^{o p} \rightarrow \mathscr{A} .
$$

Usually, we put

$$
\phi(M(E)(-n)[-n])=\phi_{n}(E)
$$

\footnotetext{
${ }^{9}$ Indeed, when $\mathscr{A}$ is the category of abelian groups, it is proved in Dég08, th. 5.1.1] that such a functor defines a cycle premodule in the sense of M. Rost.
} 
so that $\phi$ becomes a graded functor on function fields. In view of the description of the functoriality of generic motives recalled in 1.9 , $\phi$ is equipped with the following structural maps:

(1) For any extension of function fields, $\varphi: E \rightarrow L$, a corestriction $\varphi_{*}$ : $\phi_{*}(E) \rightarrow \phi_{*}(L)$ of degree 0.

(2) For any finite extension of function fields, $\varphi: E \rightarrow L$, a restriction $\varphi^{*}$ : $\phi_{*}(L) \rightarrow \phi_{*}(E)$ of degree 0 , also denoted by $N_{L / E}$.

(3) For any function field $E, \phi_{*}(E)$ admits a $K_{*}^{M}(E)$-graded module structure.

(4) For any valued function field $(E, v)$ with ring of integers essentially of finite type over $k$ and residue field $\kappa(v)$, a residue $\partial_{v}: \phi_{*}(E) \rightarrow \phi_{*}(\kappa(v))$ of degree -1 .

Definition 2.3. For any pair of integers $(q, n)$, we associate with the realization functor $H$ a cycle premodule $\hat{H}^{q, n}$ as the restriction of the functor $\bar{H}(?(n)[p])$ to the category $D M_{g m}^{(0)}(k)$, using notation (2.1.a).

According to formula (2.2.a), cycle modules are $\mathbb{Z}$-graded. This motivates the following redundant notation for a smooth scheme $X$ and a triple of integers $(q, n, r)$ :

$$
H_{r}^{q, n}(X):=H^{q+r, n+r}(X) .
$$

Note that we thus get a twisted cohomology $H_{*}^{* *}$ with values in the $\mathbb{Z}$-graded category $\mathscr{A}^{\mathbb{Z}}$. Moreover, we obtain using the motivic coniveau exact couple a spectral sequence of the form:

$$
E_{1}^{p, q}(X, n)=\bigoplus_{x \in X^{(p)}} \hat{H}_{*}^{q-p, n-p}(\kappa(x)) \Rightarrow H_{*}^{p+q, n}(X),
$$

with values in $\mathscr{A}^{\mathbb{Z}}$. Its 0 -th graded part is the spectral sequence (2.3.b).

For any function field $E$ and any triple of integers $(q, n, r)$, we accordingly write:

$$
\hat{H}_{r}^{q, n}(E)=\hat{H}^{q+r, n+r}(E)
$$

with the notation of formula (2.1.b).

Remark 2.4. Taking care of the canonical grading on cycle modules, the family of cycle modules defined above comes in with the following relation:

$$
\forall a \in \mathbb{Z}, \hat{H}_{*}^{q+a, n+a}=\hat{H}_{*-a}^{q, n} .
$$

2.5. Recall the aim of the axioms of a cycle module is to build a complex (cf Ros96. (2.1)]). We recall these axioms to the reader using the morphisms introduced in Paragraph 1.14. We say that a cycle premodule $\phi$ is a cycle module if the following two conditions are fulfilled :

(FD) Let $X$ be a normal scheme essentially of finite type over $k, \eta$ its generic point and $E$ its functions field. Then for any element $\rho \in \phi_{i}(E), \phi\left(\partial_{x}^{\eta}\right)(\rho)=0$ for all but finitely many points $x$ of codimension 1 in $X$.

(C) Let $X$ be an integral local scheme essentially of finite type over $k$ and of dimension 2. Let $\eta$ (resp. $s$ ) be its generic (resp. closed) point, and $E$ (resp. $\kappa$ ) be its function (resp. residue) field. Then, for any integer $n \in \mathbb{Z}$, the morphism

$$
\sum_{x \in X^{(1)}} \phi_{n-1}\left(\partial_{s}^{x}\right) \circ \phi_{n}\left(\partial_{x}^{\eta}\right): \phi_{n}(E) \rightarrow \phi_{n-2}(\kappa),
$$

well defined under (FD), is zero. 
When these conditions are fulfilled, for any scheme $X$ essentially of finite type over $k$, Rost defines in Ros96, (3.2)] a graded complex of cycles with coefficients in $\phi$ whose $i$-th graded $p$-cochains are

$$
C^{p}(X ; \phi)_{i}=\bigoplus_{x \in X^{(p)}} \phi_{i-p}(\kappa(x))
$$

and with $p$-th differential equal to the well defined morphism

$$
d^{p}=\sum_{(x, y) \in X^{(p)} \times X^{(p+1)}} \phi\left(\partial_{x}^{y}\right) .
$$

The cohomology groups of this complex are called the Chow groups with coefficients in $\phi$ and denoted by $A^{*}(X ; \phi)$ in [Ros96]. Actually, $A^{*}(X ; \phi)$ is bigraded according to the bigraduation on $C^{*}(X ; \phi)$.

2.6. Consider the cycle module $\hat{H}_{*}^{q, n}$ introduced in Definition 2.3 with its $\mathbb{Z}$ graduation given by formula (2.2.a). According to this definition, the $E_{1}$-term of the spectral sequence (2.3.b) can be written as:

$$
E_{1}^{p, q}(X, n)=C^{p}\left(X, \hat{H}_{*}^{q, n}\right)
$$

if we use the formula (2.5.a) for the right hand side. Moreover, according to Proposition 1.15, the differential $d_{1}^{p, q}$ of the spectral sequence is given by the formula:

$$
d_{1}^{p, q}=\sum_{(x, y) \in X^{(p)} \times X^{(p+1)}} \hat{H}_{*}^{q, n}\left(\partial_{y}^{x}\right) .
$$

This is precisely the formula $(2.5 . \mathrm{b})$ for the cycle premodule $\hat{H}^{q, n}$. Thus proposition loc. cit. implies in particular that this morphism is well defined. Moreover it shows that for any integer $r$, the graded abelian group $C^{*}\left(X, \hat{H}_{r}^{q, n}\right)$ together with the well defined differentials of the form (2.5.b) is a complex. We deduce from this fact the following proposition:

Proposition 2.7. Consider the previous notations.

(i) For any integer $q \in \mathbb{Z}$, the cycle premodule $\hat{H}_{*}^{q, n}$ is a cycle module.

(ii) For any smooth scheme $X$ and any couple $(q, n)$ of integers, there is an equality of complexes:

$$
E_{1}^{*, q}(X, n)=C^{*}\left(X ; \hat{H}_{*}^{q, n}\right),
$$

where the left hand side is the complex made by the q-th line of the first page of the spectral sequence (2.3.b).

Proof. Point (ii) follows from Preliminary 2.6 .

We prove point (i), axiom (FD). Consider a normal scheme $X$ essentially of finite type over $k$. We can assume it is affine of finite type. Then there exists a closed immersion $X \stackrel{i}{\rightarrow} \mathbb{A}_{k}^{r}$ for an integer $r \geq 0$. According to the preliminary discussion of Paragraph 2.6. $C^{*}\left(\mathbb{A}_{k}^{r} ; \hat{H}_{*}^{q, n}\right)$ is a well defined complex. Thus, axiom (FD) for the cycle premodule $\hat{H}_{*}^{q, n}$ follows from the fact the immersion $i$ induces an inclusion

$$
\hat{H}_{*}^{q, n}(E) \subset C^{r}\left(\mathbb{A}_{k}^{r} ; \hat{H}_{*}^{q, n}\right)
$$

and the definition of the differentials given above.

For axiom (C), we consider an integral local scheme $X$ essentially of finite type over $k$ and of dimension 2 . We have to prove that $C^{*}\left(X ; \hat{H}_{*}^{q, n}\right)$ is a complex - the

10 This graduation follows the convention of [Ros96 §5] except for the notation. The notation $C^{p}(X ; \phi, i)$ used by Rost would introduce a confusion with twists. 
differentials are well defined according to (FD). To this aim, we can assume $X$ is affine of finite type over $k$. Then, there exists a closed immersion $X \rightarrow \mathbb{A}_{k}^{r}$. From the definition given above, we obtain a monomorphism

$$
C^{p}\left(X ; \hat{H}_{*}^{q, n}\right) \rightarrow C^{p}\left(\mathbb{A}_{k}^{r} ; \hat{H}_{*}^{q, n}\right)
$$

which is compatible with differentials. Thus the conclusion follows again from the preliminary discussion of Paragraph 2.6.

Remark 2.8. This proposition gives a direct proof of Dég08, Th. 6.2.1].

Corollary 2.9. Using the notations of the previous proposition, the $E_{2}$-terms of the coniveau spectral sequence (2.3.b) are :

$$
E_{2}^{p, q}(X, n)=A^{p}\left(X ; \hat{H}_{*}^{q, n}\right) \Rightarrow H_{*}^{p+q, n}(X) .
$$

Moreover, for any couple of integers $(q, n)$ and any smooth proper scheme $X$, the term $E_{2}^{0, q}(X, n)$ is a birational invariant of $X$.

The second assertion follows from [Ros96, 12.10].

Example 2.10. Consider the functor $H_{\mathcal{M}}=\operatorname{Hom}_{D M_{g m}(k)}(., \mathbb{Z})$, corresponding to motivic cohomology. In this case, following [SV00, 3.2, 3.4], for any function field $E$,

$$
H_{\mathcal{M}}^{q}(E ; \mathbb{Z}(p))= \begin{cases}0 & \text { if } q>p \text { or } p<0 \\ K_{p}^{M}(E) & \text { if } q=p \geq 0\end{cases}
$$

In particular, from Definition 2.3. $\hat{H}_{\mathcal{M}}^{n, n}=K_{*+n}^{M}$. In fact, this is an isomorphism of cycle modules. For the norm, this is loc. cit. 3.4.1. For the residue, it is sufficient (using for example [Ros96, formula (R3f)]) to prove that for any valued function field $(E, v)$ with uniformizing parameter $\pi, \partial_{v}(\pi)=1$ for the cycle module $\hat{H}_{\mathcal{M}}^{n, n}$. This follows from [Dég08, 2.6.5] as for any morphism of smooth connected schemes $f: Y \rightarrow X$, the pullback $f^{*}: H_{\mathcal{M}}^{0}(X ; \mathbb{Z}) \rightarrow H_{\mathcal{M}}^{0}(Y, \mathbb{Z})$ is the identity of $\mathbb{Z}$.

As remarked by Voevodsky at the very beginning of his theory, the vanishing mentioned above implies that the coniveau spectral sequence for $H_{\mathcal{M}}$ satisfies $E_{1}^{p, q}(X, n)=0$ if $p>n$ or $q>n$. In particular, the spectral sequence gives an isomorphism: $A^{n}\left(X ; \hat{H}^{n, n}\right)_{0} \rightarrow H_{\mathcal{M}}^{2 n}(X ; \mathbb{Z}(n))$. The left hand side is $A^{n}\left(X ; K_{*}^{M}\right)_{n}$ which is nothing else than the Chow group $C H^{n}(X)$ of cycles modulo rational equivalence. This is precisely the proof of the isomorphism of Voevodsky:

$$
H_{\mathcal{M}}^{2 n}(X, \mathbb{Z}(n))=C H^{n}(X) .
$$

2.11. In the sequel, we will need the following functoriality of the Chow group of cycles with coefficients in a cycle module $\phi$ :

- $A^{*}(. ; \phi)$ is contravariant for flat morphisms ([Ros96, (3.5)]).

- $A^{*}(. ; \phi)$ is covariant for proper morphisms ([Ros96, (3.4)]).

- For any smooth scheme $X, A^{*}(X ; \phi)$ is a graded module over $C H^{*}(X)$ (Dég06, 5.7 and 5.12]).

- $A^{*}(. ; \phi)$ is contravariant for morphisms between smooth schemes ( Ros96, $\S 12])$.

Note that any morphism of cycle modules gives a transformation on the corresponding Chow group with coefficients which is compatible with the functorialities listed above. Moreover, identifying $A^{p}\left(. ; K_{*}^{M}\right)_{p}$ with $C H^{p}($.$) , following the preceding ex-$ ample, the structures above correspond to the usual structures on the Chow group. 
Finally, let us recall that the maps appearing in the first three points above are defined at the level of the complexes $C^{*}(. ; \phi)$ (introduced in 2.5).

In [BO74, the authors expressed the $E_{2}$-term of the coniveau spectral sequence as the Zariski cohomology of a well defined sheaf. We get the same result in the motivic setting. Let $\mathcal{H}_{*}^{q, n}$ be the presheaf of graded abelian groups on the category of smooth schemes such that

$$
\left.\Gamma\left(X ; \mathcal{H}_{*}^{q, n}\right)\right):=A^{0}\left(X ; \hat{H}_{*}^{q, n}\right) .
$$

Classically, this group is called the $n$-th twisted unramified cohomology of $X$ with coefficients in $H$.

Proposition 2.12. Consider the notations above.

(1) The presheaf $\mathcal{H}_{*}^{q, n}$ has a canonical structure of a homotopy invariant sheaf with transfers.

(2) There is a isomorphism of abelian groups:

$$
A^{p}\left(X ; \hat{H}_{*}^{q, n}\right)=H_{\mathrm{Zar}}^{p}\left(X ; \mathcal{H}_{*}^{q, n}\right)
$$

which is natural with respect to contravariant functoriality in the smooth scheme $X$.

Proof. The first assertion follows from [Ros96, (8.6)] and [Dég06, 6.9] while the second one follows from [Ros96, (2.6)].

2.13. Using the notations of the previous proposition, we have obtained the following form of the $\mathbb{Z}$-graded spectral sequence (2.3.b):

$$
E_{2}^{p, q}(X, n)=H_{\mathrm{Zar}}^{p}\left(X ; \mathcal{H}_{*}^{q, n}\right) \Rightarrow H_{*}^{p+q, n}(X) .
$$

This is the analog of the Corollary 6.3 of BO74] except for the definition of the sheaf $\mathcal{H}_{*}^{q, n}$. However, using the argument of loc. cit., we can recover the form considered by Bloch and Ogus for the sheaf $\mathcal{H}_{*}^{q, n}$. Indeed, the spectral sequence (2.3.b) is natural with respect to open immersions. Thus, it can be sheafified for the Zariski topology and we obtain a spectral sequence of Zariski sheaves with coefficients in $\mathscr{A}^{\mathbb{Z}}$, converging to the Zariski sheaf $\tilde{\mathcal{H}}_{*}^{q, n}$ associated with the presheaf:

$$
X \mapsto H_{*}^{q, n}(X) .
$$

According to the preceding computation of the $E_{2}$-term, we obtain that the sheafified spectral sequence is concentrated in the line $p=0$ from $E_{2}$ on. This gives an isomorphism $\tilde{\mathcal{H}}_{*}^{q, n} \simeq \mathcal{H}_{*}^{q, n}$ as required. Let us state this:

Proposition 2.14. The sheaf $\mathcal{H}_{*}^{q, n}$ defined by formula (2.11.a) is equal to the Zariski sheaf on $\mathscr{S} m_{k}$ associated with $H_{*}^{q, n}$.

Example 2.15. In CD09, Cisinski and the author have introduced axioms on a presheaf of differential graded algebras $E$ over smooth affine schemes which guarantee the existence of a realization functor

$$
H_{E}: D M_{g m}(k)^{o p} \rightarrow K-v s
$$

such that for any smooth affine scheme $X, H_{E}(M(X)[-i])=H^{i}(E(X))$. We call $E$ a mixed Weil theory. A distinctive feature of the resulting cohomology is that it is periodic with respect to the twist ; in other words, there exists an isomorphism:

$$
\epsilon_{M}: H_{E}(M(1)) \rightarrow H_{E}(M)
$$

which can be chosen to be natural in $M$.

Let us summarize the properties obtained previously for this particular kind of realization: 
- There exists canonical cycle modules $\hat{H}_{E, *}^{q, n}$ such that for any function field $L / k$,

$$
\hat{H}_{E, r}^{q, n}(L):=\lim _{A / k} H^{q+r, n+r}(\operatorname{Spec}(A))
$$

where the limit runs over the models of $L / k$ - see Par. 1.7. In fact, this family of cycle modules is equivalent to only one of them according to the following isomorphism:

$$
\hat{H}_{E, *}^{q, n}=\hat{H}_{E, *-q}^{0, n-q} \simeq \hat{H}_{E, *-q}^{0,0}
$$

where the equality follows from Remark 2.4 and the isomorphism is induced by $\epsilon$.

- Let $\mathcal{H}_{E, *}^{q, n}$ be the Zariski sheaf on $\mathscr{S} m_{k}$ associated with the presheaf $H_{E, *}^{q, n}$. Then $\mathcal{H}_{*}^{q, n}$ has a canonical structure of a homotopy invariant sheaf with transfers. Moreover, $\mathcal{H}_{*}^{q, n}(X)$ is constant on the birational class of a smooth proper scheme $X$.

There exists a spectral sequence associated with the cohomology $H_{E}$, converging to its coniveau filtration (recall formula (2.1.d)), of the following form:

$$
E_{2}^{p, q}(X, n)=A^{p}\left(X, \hat{H}_{E, *}^{q, n}\right)=H_{\mathrm{Zar}}^{p}\left(X, \mathcal{H}_{E, *}^{q, n}\right) \Rightarrow H_{E, *}^{p+q, n}(X) .
$$

Remark 2.16. Assume $k$ is a perfect field of characteristic $p>0$, let $W$ be the associated Witt ring and denote by $K$ the fraction field of $W$. According to CD07, sec. 3.2], there exists a mixed Weil theory $E$ such that:

- for any smooth affine scheme $X, H_{E}^{n}(X)$ is the Monsky-Washnitzer cohomology (of a lift of $X$ over $W$ ) - see [MW68.

- for any smooth proper scheme $X, H_{E}^{n}(X)$ is the crystalline cohomology of $X / W$ tensored with $K$.

Then, the preceding example applied to this mixed Weil theory, together with forthcoming Corollary 2.25 gives the results stated in the end of the introduction.

2.17. Regulators.- Consider again the situation and notations of the previous example. The algebra structure on $E$ induces an algebra structure on $H_{E}^{* *}$. The unit of this structure $1 \in H_{E}^{0,0}(\operatorname{Spec}(k))$ corresponds to an element $\rho \in H_{E}(\mathbb{Z})$ which induces a natural transformation

$$
\rho: H_{\mathcal{M}} \rightarrow H_{E}
$$

because motivic cohomology corresponds to the functor $\operatorname{Hom}_{D M_{g m}(k)}(?, \mathbb{Z})$. This is the regulator map - or rather, its extension to the full triangulated category of mixed motives $D M_{g m}(k)$. According to the preceding construction, this map induces a natural transformation of cycle modules:

$$
\hat{H}_{\mathcal{M}, *}^{q, n} \rightarrow \hat{H}_{E, *}^{q, n}
$$

corresponding to what should be called higher symbols. Indeed, in the case $q=n$, it gives usual symbols for any function field $L$ :

$$
K_{n}^{M}(L) \rightarrow \hat{H}_{E}^{n, n}(L) .
$$

This higher symbol map is of course compatible with all the structures of a cycle module: corestriction, restriction, residues. 


\subsection{Algebraic equivalence.}

2.18. In this section, we assume $\mathscr{A}$ is the category of $K$-vector spaces for a given field $K$.

Consider the cycle modules $\hat{H}_{*}^{q, n}$ associated with $H$ in Definition 2.3, together with their $\mathbb{Z}$-grading defined by formula (2.3.c). We introduce the following properties on the realization functor $H$ :

(Vanishing) For any function field $E$ and any couple of negative integers $(q, n)$, $\hat{H}_{0}^{q, n}(E)=0$.

(Rigidity) The covariant functor $\hat{H}_{0}^{0,0}$ on function fields over $k$ is the constant functor with value $K$.

Let us assume $H$ satisfies (Rigidity). Then the unit element of the field $K$ determines an element $\rho$ of $H(\mathbb{Z})$ through the identification

$$
K=\hat{H}_{0}^{0,0}(k)=H(\mathbb{Z}) .
$$

We deduce from $\rho$, as in example 2.15, a canonical natural transformation of contravariant functors on $D M_{g m}(k)$ :

$$
\rho: \operatorname{Hom}_{D M_{g m}(k)}(., \mathbb{Z}) \rightarrow H .
$$

Of course, the source functor is nothing else than the realization functor which corresponds to motivic cohomology $H_{\mathcal{M}}$. In particular, taking of Voevodsky's isomorphism recalled in (2.10.b), we get a canonical cycle class:

$$
\rho_{X}^{n}: C H^{n}(X)_{K} \rightarrow H^{2 n, n}(X) .
$$

Let us denote by $Z^{n}(X, K)$ the group of $n$-codimensional $K$-cycles in $X$ (simply called cycles in what follows) and by $\mathcal{K}_{\text {rat }}^{n}(X)$ (resp. $\left.\mathcal{K}_{\text {alg }}^{n}(X)\right)$ its subgroup formed by cycles rationally (resp. algebraically) equivalent to 0 .

Definition 2.19. Using the notations above, we define the group of cycles $H$ equivalent to 0 as:

$$
\mathcal{K}_{H}^{n}(X)=\left\{\alpha \in Z^{n}(X, K) \mid \rho_{X}^{n}(\alpha)=0\right\} .
$$

Remark 2.20. The map (2.18.a) induces a morphism of cycle modules $K_{*+a}^{M} \rightarrow \hat{H}_{*}^{a, a}$ which corresponds to cohomological symbols $K_{a}^{M}(E) \rightarrow \hat{H}^{a, a}(E)$ compatible with corestriction, restriction, residues and the action of $K_{*}^{M}(E)$.

2.21. We analyze the coniveau spectral sequence (2.1.c) under the assumptions (Vanishing) and (Rigidity). The $E_{1}$-term is described by the following picture:

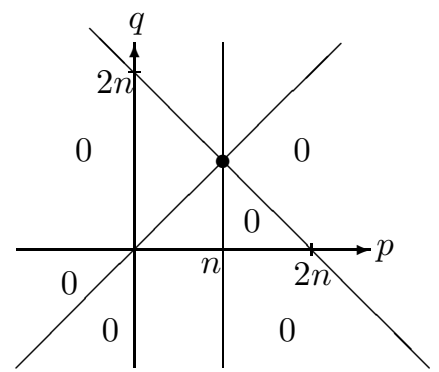

Property (Rigidity) implies that $E_{1}^{n, n}(X, n)=Z^{n}(X, K)$. As only one differential goes to $E_{r}^{n, n}$, we obtain a sequence of epimorphisms:

$$
Z^{n}(X, K)=E_{1}^{n, n}(X, n) \rightarrow E_{2}^{n, n}(X, n) \rightarrow E_{3}^{n, n}(X, n) \rightarrow \ldots
$$


which become isomorphisms as soon as $r>n$. Thus, if we put

$$
\mathcal{K}_{(r)}^{n}(X)=\operatorname{Ker}\left(E_{1}^{n, n}(X, n) \rightarrow E_{r+1}^{n, n}(X, n)\right),
$$

we obtain an increasing filtration on $Z^{n}(X, K)$ :

$$
\mathcal{K}_{(1)}^{n}(X) \subset \mathcal{K}_{(2)}^{n}(X) \subset \ldots \subset \mathcal{K}_{(n)}^{n}(X) \subset Z^{n}(X, K)
$$

such that $E_{r}^{n, n}(X, n)=Z^{n}(X, K) / \mathcal{K}_{(r-1)}^{n}(X)$.

Note also that $E_{n}^{n, n}=E_{\infty}^{n, n}$ is the first step of the coniveau filtration on $H^{2 n}(X, n)$ so that we get a monomorphism

$$
\epsilon: E_{n}^{n, n}(X, n) \rightarrow H^{2 n, n}(X) .
$$

Note these considerations can be applied to the functor $\operatorname{Hom}_{D M_{g m}(k)}(., K)$ corresponding to $K$-rational motivic cohomology. In this case, according to Example 2.10, the $E_{r}^{n, n}=C H^{n}(X)_{K}=H_{\mathcal{M}}^{2 n}(X ; K(n))$.

Returning to the general case, the natural transformation $\rho$ induces a morphism of the coniveau spectral sequences. This induces the following commutative diagram:

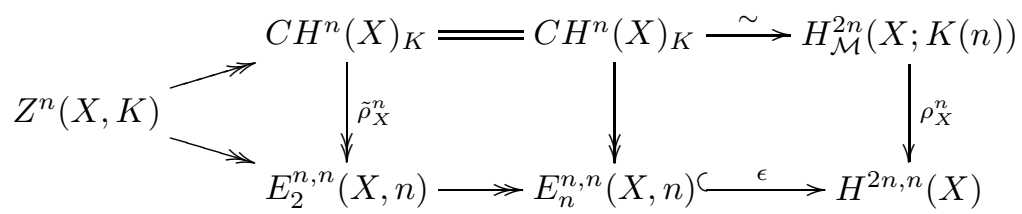

The following proposition is a generalization of a result of Bloch-Ogus (cf BO74, $(7.4)])$.

Proposition 2.22. Consider the preceding hypothesis and notations. Then the following properties hold:

(i) For any scheme $X$ and any integer $n \in \mathbb{N}, \mathcal{K}_{\text {rat }}^{n}(X) \subset \mathcal{K}_{(1)}^{n}(X)$.

(ii) For any scheme $X$ and any integer $n \in \mathbb{N}, \mathcal{K}_{(n)}^{n}(X)=\mathcal{K}_{H}^{n}(X)$.

Moreover, the following conditions are equivalent:

(iii) For any smooth proper scheme $X, \mathcal{K}_{H}^{1}(X)=\mathcal{K}_{\text {alg }}^{1}(X)$.

(iii') For any smooth proper scheme $X$ and any $n \in \mathbb{N}, \mathcal{K}_{(1)}^{n}(X)=\mathcal{K}_{\text {alg }}^{n}(X)$.

Note that under the equivalent conditions (iii) and (iii'), the morphism $\tilde{\rho}_{X}^{n}$ induces, according to (2.13.a), an isomorphism:

$$
A^{n}(X)_{K} \stackrel{\sim}{\longrightarrow} H_{\text {Zar }}^{n}\left(X ; \mathcal{H}^{n, n}\right)
$$

where $\mathcal{H}^{n, n}$ is the Zariski sheaf associated with $H^{n, n}-$ apply propositions 2.12 and 2.14 .

Proof. Properties (i) and (ii) are immediate consequences of (2.21.b).

Note that, for $n=0$, condition (iii') always holds. Obviously (iii) implies (iii') according to assertion (ii). Thus it remains to prove that (iii) implies (iii') when $n>1$.

Fix an integer $n>1$. We first prove the inclusion $\mathcal{K}_{\text {alg }}^{n}(X) \subset \mathcal{K}_{(1)}^{n}(X)$. Consider cycles $\alpha, \beta \in Z^{n}(X, K)$ such that $\alpha$ is algebraically equivalent to $\beta$. This means there exists a smooth proper connected curve $C$, points $x_{0}, x_{1} \in C(k)$, and a cycle $\gamma$ in $Z^{n}(X \times C, K)$ such that $f_{*}\left(g^{*}\left(x_{0}\right) \cdot \gamma\right)=\alpha, f_{*}\left(g^{*}\left(x_{1}\right) \cdot \gamma\right)=\beta$ where $f: X \times C \rightarrow X$ and $g: X \times C \rightarrow X$ are the canonical projections. Using the functoriality described 
in paragraph 2.11 applied to the morphism of cycle modules $K_{*}^{M} \rightarrow \hat{H}_{*}^{0,0}$ (Remark 2.20), we get a commutative diagram

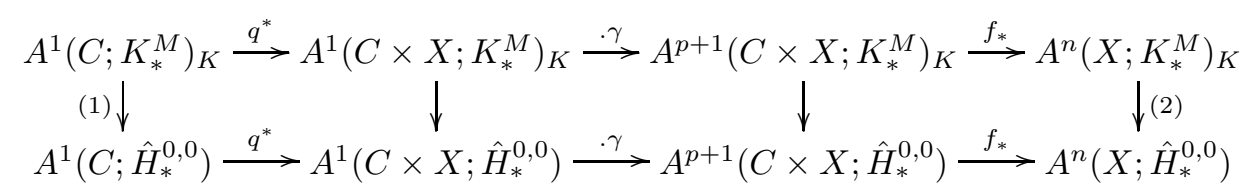

Recall the identifications:

$$
A^{n}\left(X ; K_{*}^{M}\right)_{n}=C H^{n}(X), \quad A^{n}\left(X ; \hat{H}_{*}^{0,0}\right)_{n}=A^{n}\left(X ; \hat{H}_{*}^{n, n}\right)_{0}=E_{2}^{n, n}(X, n) .
$$

According to these ones, the first (resp. $n$-th) graded piece of the map (1) (resp. (2)) can be identified with the morphism $\tilde{\rho}_{X}^{1}$ (resp. $\tilde{\rho}_{X}^{n}$ ). In particular, we are reduced to prove that $x_{0}-x_{1}$ belongs to $\mathcal{K}_{(1)}^{1}(C)$. This finally follows from (iii).

We prove conversely that $\mathcal{K}_{(1)}^{n}(X) \subset \mathcal{K}_{\text {alg }}^{n}(X)$. Recall $A^{n}\left(X ; \hat{H}_{*}^{n, n}\right)_{0}$ is the cokernel of the differential (2.5.b)

$$
C^{n-1}\left(X ; \hat{H}_{*}^{n, n}\right)_{0} \stackrel{d^{n-1}}{\longrightarrow} C^{n}\left(X ; \hat{H}_{*}^{n, n}\right)_{0}=Z^{n}(X, K) .
$$

We have to prove that the image of this map consists of the cycles algebraically equivalent to zero. Consider a point $y \in X^{(p-1)}$ with residue field $E$ and an element $\rho \in \bar{H}^{1,1}(E)$. Let $i: Y \rightarrow X$ be the immersion of the reduced closure of $y$ in $X$.

Using De Jong's theorem, we can consider an alteration $Y^{\prime} \stackrel{f}{\rightarrow} Y$ such that $Y^{\prime}$ is smooth over $k$. Let $\varphi: E \rightarrow L$ be the extension of function fields associated with $f$. According to the basic functoriality of cycle modules recalled in Paragraph 2.11 we obtain a commutative diagram

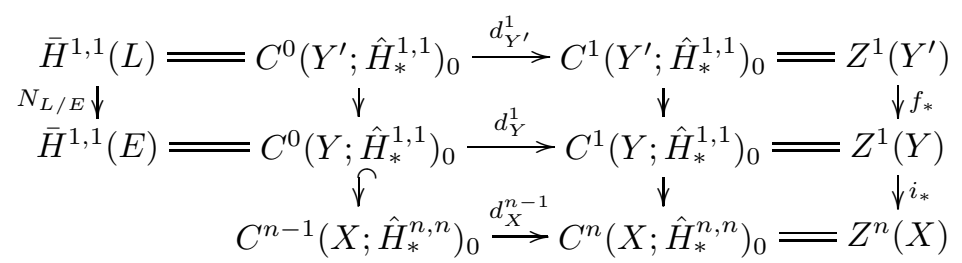

where $f_{*}$ and $i_{*}$ are the usual proper pushouts on cycles. Recall from Ros96, (R2d)] that $N_{L / E} \circ \varphi_{*}=[L: E] . I d$ for the cycle module $\hat{H}_{*}^{1,1}$. Thus, $N_{L / E}$ is surjective. As algebraically equivalent cycles are stable by direct images of cycles, we are reduced to the case of the scheme $Y^{\prime}$, in codimension 1, already obtained above.

Remark 2.23. In the preceding proof, if we can replace the alteration $f$ by a (proper birational) resolution of singularities, then the theorem is true with integral coefficients - indeed, the extension $L / E$ which shows up in the end of the proof is trivial when $f$ is birational. This holds in characteristic 0 by Hironaka's resolution of singularities but also in characteristic $p>0$ if $X$ is a curve, a surface (cf Lip78) or a 3 -fold (cf [CP09]).

2.24. We consider the assumptions and notations of Example 2.15, An important property of a mixed Weil theory is the fact the graded functor:

$$
H_{E}^{*}: D M_{g m}(k)^{o p} \rightarrow(K-v s)^{\mathbb{Z}}, M \mapsto\left(H_{E}^{n}(M), n \in \mathbb{Z}\right)
$$

is monoidal where the target category is the monoidal category of $\mathbb{Z}$-graded vector spaces.

Recall from [Dég11, Prop. 2.18] that for any smooth projective scheme $X$ of pure dimension $d$, there exists a strong duality pairing $\eta: M(X) \otimes M(X)(-d)[-2 d] \rightarrow \mathbb{Z}$. 
Applying to this pairing the monoidal functor $H_{E}^{*}$, we get the usual Poincaré duality pairing:

$$
H_{E}^{q, n}(X) \otimes H_{E}^{2 d-q, d-n}(X) \rightarrow K .
$$

As in the above, the regulator map induces a morphism of the unramified sheaves $\mathcal{H}_{M}^{q, n} \rightarrow \mathcal{H}_{E}^{q, n}$ which induces an "unramified" regulator:

$$
\tilde{\rho}_{X}: C H^{n}(X) \simeq H_{\text {Zar }}^{n}\left(X, \mathcal{H}_{\mathcal{M}}^{n, n}\right) \rightarrow H_{\text {Zar }}^{n}\left(X, \mathcal{H}_{E}^{n, n}\right)
$$

As a corollary of the preceding proposition, we get the following result:

Corollary 2.25. Consider the notations above. For any pair of integers $(q, n)$, let $\mathcal{H}_{E}^{q, n}$ be the Zariski sheaf on the category of smooth schemes associated with $H_{E}^{q, n}$.

Assume the realization functor $H_{E}$ satisfies property (Vanishing) (\$2.18). Then, the following conditions are equivalent:

(i) The realization functor $H_{E}$ satisfies property (Rigidity) (\$2.18).

(ii) For any integer $n \in \mathbb{N}$ and any projective smooth scheme $X$, the unramified regulator map $\tilde{\rho}_{X}$ considered above induces an isomorphism

$$
A^{n}(X)_{K} \rightarrow H_{\text {Zar }}^{n}\left(X ; \mathcal{H}_{E}^{n, n}\right) .
$$

Proof. Remark the assumption implies that for any smooth scheme $X$ and any $i<0, H^{i}(X, \mathbb{E})=0$ - apply the coniveau spectral sequence for $X$.

$(i) \Rightarrow(i i)$ : According to our hypothesis, we can apply Proposition 2.22 to the realization functor $H_{E}$. Indeed, we have assumed (Vanishing) and (Rigidity). Moreover, Property (Rigidity) and the Poincaré duality pairing implies that for any smooth projective connected curve $p: C \rightarrow \operatorname{Spec}(k)$, the morphism $p_{*}$ : $H^{2}(C, \mathbb{E})(1) \rightarrow H^{0}(C, \mathbb{E})=K$ is an isomorphism. Following classical arguments, this together with the multiplicativity of the cycle class map implies that homological equivalence for $\mathbb{E}$ is between rational and numerical equivalence. From Matsusaka's theorem (cf Mat57), these two equivalences coincide for divisors. This implies assumption (iii) of Proposition 2.22, and we can conclude from the isomorphism (2.22.a).

$($ ii $) \Rightarrow(i)$ : For a $d$-dimensional smooth projective connected scheme $X$, we deduce from the coniveau spectral sequence and Poincaré duality that $E_{2}^{d, d}(X, d)=$ $H^{2 d}(X, \mathbb{E})(d)=H^{0}(X, \mathbb{E})$. Thus property (ii) implies $H^{0}(X, \mathbb{E})=K$. If $L$ is the function field of $X$, we deduce that $\bar{H}^{0}(L, \mathbb{E})=K$. Considering any function field $E$, we easily construct an integral projective scheme $X$ over $k$ with function field $E$. Applying De Jong's theorem, we find an alteration $\tilde{X} \rightarrow X$ such that $\tilde{X}$ is projective smooth and the function field $L$ of $\tilde{X}$ is a finite extension of $E$ and the result now follows from the fact $N_{L / E}: \bar{H}^{0}(L) \rightarrow \bar{H}^{0}(E)$ is a split epimorphism.

Remark 2.26. (1) Condition (i) in the previous corollary is only reasonable when the base field $k$ is separably closed (or after an extension to the separable closure of $k$ ).

(2) When $k$ is the field of complex numbers and $H$ is algebraic De Rham cohomology, the filtration on cycles (2.21.a) is usually called the BlochOgus filtration - see [Fri95. It can be compared with other filtrations (see Nor93, Fri95). It is an interesting question whether a similar comparison to that of [Nor93, rem. 5.4] can be obtained in the case of rigid cohomology.

\section{REFERENCES}

[Ber74] P. Berthelot. Cohomologie cristalline des schémas de caractéristique $p>0$. SpringerVerlag, Berlin, 1974. Lecture Notes in Mathematics, Vol. 407. 
[Ber97] P. Berthelot. Finitude et pureté cohomologique en cohomologie rigide. Invent. Math., 128:329-377, 1997.

[BO74] S. Bloch and A. Ogus. Gersten's conjecture and the homology of schemes. Ann. Sci. École Norm. Sup. (4), 7:181-201 (1975), 1974.

[CD07] D.-C. Cisinski and F. Déglise. Mixed Weil cohomologies. arXiv:0712.3291, 2007.

[CD09] D.-C. Cisinski and F. Déglise. Triangulated categories of mixed motives. arXiv:0912.2110, 2009.

[CP09] Vincent Cossart and Olivier Piltant. Resolution of singularities of threefolds in positive characteristic. II. J. Algebra, 321(7):1836-1976, 2009.

[CT03] B. Chiarellotto and N. Tsuzuki. Cohomological descent of rigid cohomology for étale coverings. Rend. Sem. Mat. Univ. Padova, 109:63-215, 2003.

[CTHK97] J.-L. Colliot-Thélène, R. T. Hoobler, and B. Kahn. The Bloch-Ogus-Gabber theorem. In Algebraic K-theory (Toronto, ON, 1996), volume 16 of Fields Inst. Commun., pages 31-94. Amer. Math. Soc., Providence, RI, 1997.

[Dég06] F. Déglise. Transferts sur les groupes de Chow coefficients. Mathematische Zeitschrift, 252:315-343, 2006.

[Dég08] F. Déglise. Motifs génériques. Rendiconti Sem. Mat. Univ. Padova, 119, 2008.

[Dég09] F. Déglise. Modules homotopiques. arXiv:0904.4747v2, 2009.

[Dég10] F. Déglise. Orientable homotopy modules. arXiv:1005.4187, 2010.

[Dég11] F. Déglise. Around the gysin triangle I. arXiv:0804.2415v2, 2007 (revised 2011).

[Fri95] E. M. Friedlander. Filtrations on algebraic cycles and homology. Ann. Sci. École Norm. Sup. (4), 28(3):317-343, 1995.

[FSV00] E.M. Friedlander, A. Suslin, and V. Voevodsky. Cycles, Transfers and Motivic homology theories. Princeton Univ. Press, 2000.

[Ger73] S. M. Gersten. Problems about higher K-functors. In Algebraic K-theory, I: Higher K-theories (Proc. Conf., Battelle Memorial Inst., Seattle, Wash., 1972), pages 43-56. Lecture Notes in Math., Vol. 341. Springer, Berlin, 1973.

[Gro66] A. Grothendieck. On the de Rham cohomology of algebraic varieties. Inst. Hautes Études Sci. Publ. Math., (29):95-103, 1966.

[Gro68] A. Grothendieck. Le groupe de Brauer. III. Exemples et compléments. In Dix Exposés sur la Cohomologie des Schémas, pages 88-188. North-Holland, Amsterdam, 1968.

[Gro69] A. Grothendieck. Hodge's general conjecture is false for trivial reasons. Topology, 8:299-303, 1969.

[Har66] Robin Hartshorne. Residues and duality. Lecture notes of a seminar on the work of A. Grothendieck, given at Harvard 1963/64. With an appendix by P. Deligne. Lecture Notes in Mathematics, No. 20. Springer-Verlag, Berlin, 1966.

[Kat86] Kazuya Kato. Milnor $K$-theory and the Chow group of zero cycles. In Applications of algebraic $K$-theory to algebraic geometry and number theory, Part I, II (Boulder, Colo., 1983), volume 55 of Contemp. Math., pages 241-253. Amer. Math. Soc., Providence, RI, 1986.

[Lip78] Joseph Lipman. Desingularization of two-dimensional schemes. Ann. Math. (2), 107(1):151-207, 1978.

[Mat57] T. Matsusaka. The criteria for algebraic equivalence and the torsion group. Amer. J. Math., 79:53-66, 1957.

[McC01] J. McCleary. A user's guide to spectral sequences, volume 58 of Cambridge Studies in Advanced Mathematics. Cambridge University Press, Cambridge, second edition, 2001.

[Mil70] John Milnor. Algebraic K-theory and quadratic forms. Invent. Math., 9:318-344, 1969/1970.

[MW68] P. Monsky and G. Washnitzer. Formal cohomology: I. Annals of Math., 88:181-217, 1968.

[Nor93] M. V. Nori. Algebraic cycles and Hodge-theoretic connectivity. Invent. Math., 111(2):349-373, 1993.

[Qui73] D.G. Quillen. Higher algebraic K-theory. I. In Algebraic K-theory, I: Higher Ktheories (Proc. Conf., Battelle Memorial Inst., Seattle, Wash., 1972), pages 85-147. Lecture Notes in Math., Vol. 341. Springer, Berlin, 1973.

[Ros96] M. Rost. Chow groups with coefficients. Doc. Math. J., pages 319-393, 1996.

[SV00] A. Suslin and V. Voevodsky. Bloch-Kato conjecture and motivic cohomology with finite coefficients. In The arithmetic and geometry of algebraic cycles (Banff, AB, 1998), 
volume 548 of NATO Sci. Ser. C Math. Phys. Sci., pages 117-189. Kluwer Acad. Publ., Dordrecht, 2000.

LaGa, CNRS (UMR 7539), Université Paris 13, Avenue Jean-Baptiste Clément, 93430 Villetaneuse, France

E-mail address: deglise@math.univ-paris13.fr

URL: http://www .math.univ-paris13.fr/ deglise/ 